\title{
Meta
}

Journal des traducteurs

Translators' Journal

\section{WYLER, L. (2003) : Línguas, poetas e bacharéis - Uma crônica da tradução no Brasil, Rio de Janeiro, Rocco, 158 p.}

\section{Denise Capra de Almeida}

Volume 50, numéro 3, août 2005

Le prisme de l'histoire

The History Lens

URI : https://id.erudit.org/iderudit/011616ar

DOI : https://doi.org/10.7202/011616ar

Aller au sommaire du numéro

Éditeur(s)

Les Presses de l'Université de Montréal

ISSN

0026-0452 (imprimé)

1492-1421 (numérique)

Découvrir la revue

Citer ce compte rendu

Capra de Almeida, D. (2005). Compte rendu de [WYLER, L. (2003) : Línguas, poetas e bacharéis - Uma crônica da tradução no Brasil, Rio de Janeiro, Rocco,

158 p.] Meta, 50(3), 1056-1058. https://doi.org/10.7202/011616ar

Ce document est protégé par la loi sur le droit d'auteur. L'utilisation des services d'Érudit (y compris la reproduction) est assujettie à sa politique d'utilisation que vous pouvez consulter en ligne.

https://apropos.erudit.org/fr/usagers/politique-dutilisation/ 


\section{RÉFÉRENCES}

Menéndez Pelayo, M. (1952-1953): Biblioteca de traductores españoles, Madrid-Santander, CSIC, vol. LIV-LVII de la Edición nacional de las obras completas.

Navarro Domínguez, F. (1996) : Manual de bibliografía española de traducción e interpretación. Diez años de historia: 1985-1995, Alicante, Universidad de Alicante.

Pellicer Saforcada, J. A. (1778): Ensayo de una biblioteca de traductores españoles, Madrid, Sancha.

Рум, A. (2000) : Negotiating the Frontier: Translators and Intercultures in Hispanic History, Manchester, St. Jerome Publishing.

Ruiz Casanova, J. F. (2000): Aproximación a una historia de la traducción en España, Madrid, Cátedra.

SAntoyo, J.-C. (1996) : Bibliografía de la traducción en español, catalán, gallego y vasco, León, Universidad de León.

Wyler, L. (2003): Línguas, poetas e bacharéis - Uma crônica da tradução no Brasil, Rio de Janeiro, Rocco, 158 p.

À l'instar des autres pays de l'Amérique latine, le Brésil a commencé depuis quelques années à se pencher sur son histoire de la traduction. Des auteurs comme Paulo Ronai (1987), Else Vieira Pires (1998), John Milton (1993 et 2001), Pagano et Vasconcelos (2003) et Campos (2004) ont en effet abordé le sujet. Le présent ouvrage de Lia Wyler vient toutefois combler un vide criant. Il est en effet le premier à embrasser l'intégralité de l'histoire brésilienne de la traduction, plus précisément de 1500 à 1979. Si l'auteure se limite à un bien bref panorama de la pratique traductrice dans ce pays, elle a l'immense mérite, en pionnière, d'ouvrir de nombreuses pistes de recherche. Rappelons toutefois, avant tout chose, que Lia Wyler est désormais internationalement connue et reconnue pour ses adaptations créatives de Harry Potter. Traductrice des cinq volumes jusqu'ici publiés, Lia Wyler a recréé le jargon haripotérien pour le plus grand plaisir des Brésiliens.

Contrairement à ce qu'indique son titre, ce livre est loin d'être une chronique, expression peut-être due à la modestie de l'auteure qui n'a vu dans son livre qu'un récit synthétique. Pourtant la minutie des données et la vision de l'ensemble font état d'une recherche en profondeur.

Cet ouvrage est un exemple de vulgarisation scientifique, une synthèse habile, rédigée dans un portugais impeccable et accessible, tant pour les chercheurs intéressés que pour les non-initiés. Que Wyler se réfère à la vie culturelle et politique brésilienne, ou qu'elle expose les grands événements qui ont marqué la pratique traduisante, elle donne à son texte une direction: survoler de façon claire et concise l'histoire de la traduction au Brésil, en guise d'introduction aux travaux qui ne devraient tarder dans ce domaine encore vierge. L'ouvrage aurait pu être intitulé: Esquisse d'une histoire de la traduction au Brésil.

Chez Wyler, le caractère informatif du texte permet aux lecteurs d'acquérir une connaissance de l'histoire du Brésil, nécessaire à la compréhension d'une perspective historique du domaine de la traduction et des horizons correspondants. L'auteure reconstitue, par exemple, la naissance et la consolidation de la profession de traducteur et d'interprète au Brésil, en abordant des sujets tels que le désintérêt des Portugais pour le développement intellectuel de la colonie, la dissémination de la langue tupi, l'établissement tardif de la première maison d'édition brésilienne (1808), la domination culturelle française et les différentes formes de censure.

L’ouvrage couvre la période qui va de la découverte du Brésil par les Portugais (1500) à la reconnaissance légale de la profession de traducteur (1979). Après une brève introduction, 
six chapitres se succèdent qui semblent, par leur présentation, donner à l'ouvrage une progression chronologique; en fait, celle-ci se fait plutôt d'une manière thématique. Lia Wyler fait ainsi ressortir la non-continuité, une caractéristique de l'histoire de la traduction au Brésil.

Dans l'introduction, l'auteure aborde la traduction dans la presse et dans les universités brésiliennes, les différentes approches de la recherche historiographique au Brésil, les variables et les racines de l'invisibilité du traducteur. Le premier chapitre est consacré à l'histoire de la pratique de la traduction orale au Brésil ; y sont étudiés notamment le contexte linguistique, la définition des rôles et l'institutionnalisation de la profession d'interprète. Le deuxième chapitre porte sur la traduction écrite durant la période coloniale, c'est-à-dire de 1500 à 1808. Dans le troisième chapitre, le lecteur trouvera une présentation intéressante de la problématique de la censure et des droits d'auteur à l'égard des traductions faites au Brésil après l'installation de la cour portugaise à Rio entre 1808 et 1821. Le quatrième chapitre relate les succès de la traduction théâtrale (1779 à 1960) et des romans-feuilleton (à partir de 1839). Dans le cinquième chapitre, Wyler présente la traduction comme un reflet de la nouvelle révolution nationaliste brésilienne des années 1930. C’est dans ce même chapitre que l'auteure décrit les circonstances qui entourent l'apparition du mouvement prônant la qualité des traductions et associant art de traduire et édition (1940). Finalement, dans le dernier chapitre, Lia Wyler décrit l'histoire récente (depuis les années 1970), notamment la création de l'Association brésilienne des traducteurs, en 1974, et le projet de loi sur la reconnaissance légale de la profession de traducteur au Brésil en 1977.

Il ne faut cependant pas s'attendre à ce que chacun de ces chapitres offre l'état de la question exhaustif d'une nouvelle problématique ou d'une époque précise. L'auteure condense en effet les informations de 479 ans d'histoire dans 158 pages! Elle le fait avec objectivité, mais s'attarde ponctuellement sur quelques sujets privilégiés: la problématique de «l'invisibilité » du traducteur, la traduction orale et écrite, la filière académique de la traduction, les problèmes du marché, les politiques linguistiques et la censure, la traduction dans la presse, le roman-feuilleton et le théâtre traduits, l'industrie de la traduction, les femmes interprètes et les préjugés, la traduction dans les années «1970», entre autres.

Linguas, poetas e bacharéis - Uma crônica da tradução no Brasil est un des ouvrages les plus utiles qui aient été produits sur le sujet, ne fût-ce que pour les recherches plus poussées qu'il ne manquera pas de susciter. Chacun de ses chapitres pourrait donner lieu à des études fouillées qui contribueraient à édifier une véritable histoire de la traduction au Brésil. Les étudiants, les enseignants et les chercheurs y trouveront de précieuses références, même s'ils doivent corriger certaines imperfections de forme, en particulier quelques fautes de frappe dans les noms propres étrangers: Le Bourgeois Getilhomme (p. 96), et George Gomow, Lewis Carrol et Robison Crusoe (p. 119).

Étant donné l'étendue de la période étudiée, l'abondance d'informations et le style de chronique adopté par Wyler, un tableau synoptique aurait permis une meilleure vue d'ensemble des sujets analysés. On peut aussi regretter l'absence d'une liste des "grandes» traductions brésiliennes, notamment l'Odyssée et l'Enéide, par Odorico Mendes, Le Corbeau, par Machado de Assis, Le pape et le Concile, par Rui Barbosa, les poèmes de Baudelaire et l'Antigone, par Guilherme de Almeida, ainsi que de certains documents historiques qui ont tant influencé les mouvements historiques et culturels brésiliens. Mais, nous l'avons dit, l'ouvrage n'a aucune prétention d'exhaustivité.

Muito obrigada, Lia! 


\section{RÉFÉRENCES}

CAmpos, G. (2004): For whom the bell tolls, de Ernest Hemingway, e suas traduções no contexto brasileiro, thèse de doctorat.

Milton, J. (1993): O poder da tradução, São Paulo, Ars Poética, 194 p.

Pagano, A. et M. L. VAsconcelos (2003) : «Estudo da Tradução no Brasil : Reflexão sobre teses e dissertações elaboradas por pesquisadores brasileiros nas décadas de 1980 e 1990 », dans D.E.L.T.A. Documentação de Estudos em Linguística Teórica e Aplicada, V. 19, p. 7-11.

RónAI, P. (1987): Escola de tradutores, Rio de Janeiro, Nova Fronteira.

Milton, J. (2001) : Emerging Views on Translation History in Brazil (édition spéciale), São Paulo, Humanitas, FFLCH/USP, 286 p.

L'ouvrage est un numéro spécial de la revue $C R O P$, un périodique de la Faculté de philosophie, lettres et sciences humaines de l'Université de São Paulo, Brésil, compilé par John Milton, qu'il n'est plus nécessaire de présenter. Cette collection d'articles, tous rédigés en anglais, portant sur l'histoire de la traduction est issue du $1^{\text {er }}$ Forum international de traducteurs tenu à São Paulo en septembre 1998 et est le reflet de la vivacité et de la maturité croissantes de cette branche de la traductologie au Brésil.

Le volume est composé de neuf articles, trois comptes rendus et une interview. Huit des articles abordent des sujets brésiliens : un panorama de l'histoire de la traduction au Brésil; la traduction du théâtre brésilien; la traduction des premiers textes en langue tupi; l'histoire de la traduction de l'œuvre de Herman Melville en portugais brésilien; l'application des théories du polysystème d'Even-Zohar à la traduction de la littérature brésilienne dans d'autres littératures; les techniques d'adaptation utilisées dans la traduction de la littérature pour enfants; le parallèle entre la traduction au Brésil et en Argentine; et l'histoire du premier Club du livre brésilien.

L'article qui ouvre le recueil est le seul non brésilien. Dans «Why and how to write translation histories?», Lieven D’hulst présente les procédures et les critères qui régissent l'écriture d'une histoire de la traduction. Partant du postulat que l'historiographie doit essentiellement viser à la reconstruction du passé tel qu'il était vraiment, l'auteur propose le plus grand nombre possible de paramètres. Il les présente au lecteur d'une façon à la fois simple, originale et didactique: Quis?, Quid?, Ubi?, Quibus auxilius?, Cur?, Quomodo?, Quando? et Cui bono?

Lia Wyler signe deux articles. Le premier, «Translating Brazil», est un survol de l'histoire de la traduction au Brésil et fait partie d'une étude dans laquelle la production des traductions est examinée dans son contexte social, politique et économique. L'article souligne les différentes appellations données à la traduction au Brésil par rapport à celles rencontrées dans certains pays européens, comment les notions traductologiques ont évolué au fil du temps, et quels sont les principaux effets des traductions sur la langue et la littérature brésiliennes. Le deuxième article, "Theatre, translation and colonization", propose de montrer la prépondérance des pièces étrangères traduites et mises en scène au Brésil à l'époque où le théâtre a été introduit par les colons portugais; il expose ensuite comment cette préséance étrangère a affecté l'activité créatrice brésilienne tout au long des siècles, en moulant le travail des auteurs sur les préférences de leur public. Il indique également le dilemme auquel font face les chercheurs qui travaillent sur l'histoire pour distinguer ce qui est traduction de ce qui est original dans le développement du théâtre au Brésil.

L'article "The translation of the first texts to tupi, the classical indian language in Brazil", de Eduardo de Almeida Navarro, décrit l'influence du tupi sur la formation de la culture brésilienne, particulièrement sur le portugais brésilien, la littérature brésilienne et les 